\title{
Myocardial Infarction Type 2: Avoiding Pitfalls and Preventing Adverse Outcomes
}

\author{
Muhammad Umer Siddiqui, MD; Adnan Ahmed, MD; Muhammad Danial Siddiqui, MD;
} and Ahmed K. Pasha, MD

\begin{abstract}
Myocardial infarction type 2 (MI type 2) is an elevation of cardiac biomarkers in a physiologically stressful state leading to demand-supply mismatch of oxygen. This type of myocardial infarction is commonly seen in hospitalized patients. Since the introduction of clear definition, diagnostic criteria and International Classification of Disease (ICD) codes, the diagnosis has become increasingly common. There still remains plenty to learn about MI type 2 especially prevention and treatment strategies. Studies have shown that there is increased mortality and morbidity associated with MI type 2 when compared to $\mathrm{Ml}$ type I, and there may be benefit in having a multi-disciplinary approach including cardiology when treating such patients. Secondary prevention therapies may also play a role in decreasing adverse events from MI type 2. However, randomized control trials are insufficient, and results of studies are cautiously interpreted. In this article we have assessed the current evidence on $\mathrm{Ml}$ type 2 and the gap in literature that will potentially be the focus of future analyses.
\end{abstract}

Keywords: Cardiac abnormalities; Ventricular septal defect; Twin-twin transfusion

$\mathrm{M}$ yocardial infarction (MI) is a major cause of mortality and morbidity in the world. With the advancement in prevention and treatment strategies, the mortality and morbidity associated with MI has significantly decreased. As we continue to explore ways to further improve the outcomes associated with MI, new entities have been described in medical literature. ${ }^{1}$ One such entity is MI type 2. The 2012 task force for definition of MI described MI type 2 as "myocardial infarction secondary to ischemia due to either increased demand or decreased oxygen supply."

Though it has been several years since MI type 2 was defined, its recognition in clinical practice lags behind MI type 1. A large real-life Swedish register study included 20,138 patients with acute myocardial infarction (AMI); $7.1 \%$ of the cases were diagnosed as MI type 2. ${ }^{2}$ In another study, Chapman et $\mathrm{al}^{3}$ followed 2,122 patients admitted with elevated troponin
Corresponding Author: Muhammad Umer Siddiqui, MD, Hospital Medicine/ Internal Medicine, Marshfield Clinic, 6529 Prairie Park Dr., Eau Claire,WI 5470I Email: siddiqui.muhammad@marshfieldclinic.org
Received: April 24, 2020

Ist revision: May 21, 2020

2nd Revision: August 12, 2020

Accepted: August 15, 2020

doi: $|0.3| 2 \mid / \mathrm{cmr} .2020 .1574$ 
and among them 429 (20.2\%) were diagnosed with MI type 2. This large variation in diagnosis of MI type 2 among hospitals is perhaps due to lack of clear definition and a diagnostic criterion, as it is sometimes difficult to distinguish MI type 1 from type $2 .{ }^{1}$ However, the incidence of MI type 2 is expected to increase after the introduction of ICD codes.

Diagnosis of MI type 2 requires elevated troponin concentration (biomarker for myocardial injury), lack of signs and symptoms suggesting MI type 1 and non-ischemic contributors to myocardial injury (such as myocarditis/heart failure), and identification of physiological stressors leading to demand-supply mismatch. ${ }^{4}$ Initial treatment after diagnosis of MI type 2 usually involves management of physiological stressors causing demand-supply mismatch. In a study by Baron et al, ${ }^{2}$ inpatient coronary angiography was only performed in $36 \%$ of the cases with type $2 \mathrm{MI}$ in comparison to $77 \%$ of the patients with MI type 1 . While normal coronary arteries were more frequently seen in MI type 2 when compared to MI type $1(42.4 \%$ vs $7.4 \%)$, an invasive treatment was less common, and antiplatelet medications were less prescribed in patients with MI type $2 .^{2}$

Compared to MI type 1, patients with MI type 2 were older and had more cardiovascular risk factors including diabetes. Other co-morbidities including anemia, renal dysfunction, infection/inflammation, atrial fibrillation, and heart failure were more frequent in patients with MI type 2 as well. ${ }^{2}$ Given advanced age and more co-morbidities, patients with MI type 2 are expected to have worse prognosis. Consistent with this statement, Chapman et $\mathrm{al}^{3}$ observed that the all-cause mortality in MI type 2 was twice as high when compared to MI type 1. The major adverse cardiovascular events (MACEs) were similar between MI type 1 and type $2 .^{3}$

Timely diagnosis of MI type 2 and initiating treatment and prevention strategies will not only help in decreasing the mortality but also help in reducing the 30-day hospital readmission rate. According to Hospital Readmission Reduction Program (HRRP), hospitals can be financially penalized for a higher than standard 30-day readmission rate. Among the patients readmitted within 30 days of discharge from the hospital, $10 \%$ are estimated to be from MI type $2 .{ }^{5}$

Due to lack of clear guidelines for diagnosing MI type 2 and the inability to code until few years ago, not much research work was performed, but this is now changing. More physicians are aware of MI type 2 definition and its ICD code. Therefore, a lot more research is being conducted to improve evidence-based practice. However, several gaps remain and will be the focus of future research.

\section{Lack of Randomized Controlled Trials}

Randomized controlled trials were not possible previously due to ambiguity in defining and diagnosing. Administrative coding was difficult as well since it was not introduced until 2017. ${ }^{6}$ However, the definition is now more transparent, and availability of ICD code has made identification of subjects possible as well.

\section{Lack of a Diagnostic Tool}

With difficulty in differentiating MI type 1 from type 2 in clinical practice, perhaps a diagnostic tool may aid in diagnosis.

\section{Prescribing Secondary Prevention Therapies}

Patients with MI type 2 are less likely to receive secondary prevention therapy which includes aspirin/statin/beta-blockers when compared to MI type $1 .^{7}$ Future studies to identify and remove barriers in prescribing these medications in the clinical practice will lead to increased utilization of secondary prevention therapies in MI type 2.

\section{Multi-Disciplinary Approach}

Is there a role for multi-disciplinary approach in MI type 2? Identifying patients at high risk of coronary artery disease and consulting cardiology for possible invasive workup to identify and treat coronary artery disease may lead to improved outcomes.

\section{Risk Stratification Tool}

Making a risk stratification tool similar to TIMI or GRACE tools will help in identifying patients who are at high risk of coronary artery disease. This will in turn guide physicians on initiating secondary prevention therapies and consulting cardiology for further recommendations.

MI type 2 in patients with coronary artery disease have worse outcomes when compared to MI type $1 .^{8}$ The awareness among the medical community of MI type 2 can be improved further. The adverse outcomes may be averted by using secondary prevention techniques for coronary artery disease and prompt referral to cardiology for further evaluation and treatment. However, the evidence that secondary prevention techniques are beneficial in MI type 2 is lacking, and randomized clinical trials are needed for clear guidelines. Until then an individualized approach is suggested based on risk-benefit profile of the patient.

In conclusion, with the increasing awareness, availability of high sensitivity troponin assays and administrative coding, the diagnosis of MI type 2 is expected to continue to rise. With the increasing diagnosis, the burden on healthcare can only be averted with wide scale research especially uncovering diagnostic tools, biomarkers and predictability scores, which may help elaborate the likelihood of complications and mortality from this diagnosis. The ability to predict the need for invasive treatment or medical management is the only way to streamline resources going forward. 


\section{References}

1. Thygesen K, Alpert JS, Jaffe AS, et al; Joint ESC/ACCF/ AHA/WHF Task Force for Universal Definition of Myocardial Infarction; Authors/Task Force Members Chairpersons; Biomarker Subcommittee; ECG Subcommittee; Imaging Subcommittee; Classification Subcommittee; Intervention Subcommittee; Trials \& Registries Subcommittee; Trials \& Registries Subcommittee; Trials \& Registries Subcommittee; Trials \& Registries Subcommittee; ESC Committee for Practice Guidelines (CPG); Document Reviewers. Third universal definition of myocardial infarction. J Am Coll Cardiol. 2012;60(16):1581-1598.

2. Baron T, Hambraeus K, Sundström J, Erlinge D, Jernberg T, Lindahl B; TOTAL-AMI study group. Type 2 myocardial infarction in clinical practice. Heart. 2015;101(2):101-106.

3. Chapman AR, Shah ASV, Lee KK, et al. Long-Term Outcomes in Patients With Type 2 Myocardial Infarction and Myocardial Injury. Circulation. 2018;137(12):1236-1245.

4. McCarthy CP, Vaduganathan M, Januzzi JL Jr. Type 2 Myocardial Infarction-Diagnosis, Prognosis, and Treatment. JAMA. 2018;320(5):433-434.

5. Martin LM, Januzzi JL Jr, Thompson RW, et al. Clinical Profile of Acute Myocardial Infarction Patients Included in the Hospital Readmissions Reduction Program. J Am Heart Assoc. 2018;7(16):e009339.

6. Mills, Lorie B. Susan Sweeney, Laura Little, Abhinav Goyal. "Red Flags for Myocardial Infarctions Coding and CDI." Journal of AHIMA, no. 7 (Jul-Aug): 54-55. Available at: https://bok.ahima.org/doc?oid=302775\#.X39WS-1OlEY. Last accessed: October 8, 2020.

7. López-Cuenca A, Gómez-Molina M, Flores-Blanco PJ, et al. Comparison between type- 2 and type-1 myocardial infarction: clinical features, treatment strategies and outcomes. J Geriatr Cardiol. 2016;13(1):15-22.

8. Gupta S, Vaidya SR, Arora S, Bahekar A, Devarapally SR. Type 2 versus type 1 myocardial infarction: a comparison of clinical characteristics and outcomes with a meta-analysis of observational studies. Cardiovasc Diagn Ther. 2017;7(4):348358.

\section{Author Affiliations}

Muhammad Umer Siddiqui, MD*; Adnan Ahmed, MD $\%$;

Muhammad Danial Siddiqui, MDf; and Ahmed K. Pasha, MDई

*Hospital Medicine/Internal Medicine, Marshfield Clinic Medical Center, Rice Lake, Wisconsin USA

FInternal Medicine, Amita Health Saint Joseph Hospital, Chicago, Illinois USA

Internal Medicine, University of South Dakota, Sioux Falls, South

Dakota USA

§Internal Medicine, Mayo Clinic, Mankato, Minnesota USA 